\title{
Chronically unprepared: emergency preparedness status among US medically vulnerable populations
}

\author{
David Barbato $^{1} \cdot$ Lindsey Bryie $^{1} \cdot$ Carolina Maria Carlisle $^{1}$ (I) $\cdot$ Padydeh Doroodchi $^{1} \cdot$ Patricia Dowbiggin $^{1}$. \\ Larissa Brunner Huber ${ }^{1}$
}

Received: 17 July 2020 / Accepted: 11 February 2021 / Published online: 5 March 2021

(C) The Author(s), under exclusive licence to Springer-Verlag GmbH Germany, part of Springer Nature 2021

\begin{abstract}
Background The prevalence of large-scale natural and biological disasters has increased in recent years and can have detrimental impacts on health. Some populations are more susceptible to these impacts, including medically vulnerable populations. The purpose of this study was to evaluate the association between medically vulnerable populations and perceived emergency preparedness status.

Methods This study used 2010 and 2012 Behavioral Risk Factor Surveillance System data $(n=33,852)$. Participants were classified into four exposure groups related to medical vulnerability for each of three chronic diseases. The outcome was based on responses to a question that asked how prepared the individual's household was to handle a large-scale disaster or emergency. Logistic regression was used to assess the medical vulnerability-preparedness association.

Results In adjusted analyses, individuals who were considered medically vulnerable had approximately $40 \%$ decreased odds of feeling prepared (OR range 0.61-0.64) compared to individuals without chronic diseases and disabilities.

Conclusions Public health professionals should direct their efforts toward medically vulnerable individuals and their preparedness statuses. This study further solidifies the need for community partnerships between medical, emergency, and public health professionals to help individuals prepare for future emergencies.
\end{abstract}

Keywords Chronic diseases $\cdot$ Disabilities $\cdot$ Emergency preparedness $\cdot$ Medically vulnerable

\section{Introduction}

Natural and biological disasters are emergency events that generally impact a large geographic community of people at once (United Nations [UN] 2009). Natural disasters include earthquakes, volcanic eruptions, landslides, tsunamis, floods, and droughts, and they can cause environmental damage, social and economic disruption, property damage, loss of life, injury, or other negative health impacts (UN 2020). In 2015, more than 370 events were registered as meeting this

David Barbato, Lindsey Bryie, Carolina Maria Carlisle, Padydeh Doroodchi, Patricia Dowbiggin, and Larissa Brunner Huber contributed equally to this work.

Carolina Maria Carlisle

carolinamcarlisle@gmail.com

1 Department of Public Health Sciences, UNC Charlotte, Charlotte, NC 28223, USA definition of large-scale natural disaster emergencies (GuhaSapir et al. 2012). It was estimated that the United States (U.S.) bore more than $\$ 300$ billion in damage in 2017, which was considered the highest annual cost on record (National Oceanic and Atmospheric Administration [NOAA] 2020). Additionally, biological threats of a global outbreak of an infectious disease such as SARS, H1N1, and more recently COVID-19, have impacted the world. Globally, the COVID19 pandemic has reached the far corners of the earth with 86,532,134 known cases and 1,871,100 deaths (Johns Hopkins University and Medicine 2021). In the U.S. in 2020, over 28 million cases of COVID-19 were diagnosed and over 300,000 deaths occurred (National Center for Immunization and Respiratory Diseases [NCIRD] 2020). In addition to the morbidity and mortality associated with COVID-19, the economic disruption associated with the pandemic has been staggering with the unemployment rate in the U.S. reaching a high of nearly $15 \%$ in April 2020, the highest level since the Great Depression (Bureau of Labor Statistics 2020). As the frequency and severity of both natural and 
biological disasters continue to increase annually, adequate emergency preparedness can minimize the impact of these disasters, especially for individuals highly susceptible to harm (Smith and Notaro 2015; O'Sullivan and Phillips 2019; Shapira et al. 2020).

Diminished health status in individuals has been associated with poor outcomes in the event of an emergency, including difficulty accessing emergency services (Shapira et al. 2020; O'Sullivan and Phillips 2019; Nick et al. 2009). People with chronic health diseases, such as hypertension, diabetes, or asthma, are at an increased risk of adverse health outcomes in the face of natural and biological disasters, and this risk increases exponentially with the severity of the event (Smith and Notaro 2015; Shapira et al. 2020). In 2012, it was estimated that nearly half of all adults in the U.S. (117 million people) suffered from at least one chronic disease, and one in four U.S. adults had multiple chronic conditions (Ward et al. 2014). Despite known increased risks of morbidity associated with those chronic conditions, research has not found that individuals with chronic conditions have increased levels of preparedness (Ko et al. 2014; Shapira et al. 2020). Ko et al. (2014) investigated the relationship between chronic diseases and general emergency preparedness in the U.S. They reported that despite individuals with chronic conditions needing specialized medical needs after a disaster, they were not better prepared for such events compared to those without chronic diseases.

Outside of medical chronic diseases, it is estimated that one in five Americans (approximately 53 million people) has some type of disability (i.e., visually impaired, hard-of-hearing) that makes it difficult for them to carry out daily activities (Centers for the Disease Control and Prevention [CDC] 2015). Smith and Notaro (2015) examined general preparedness for emergencies among persons with disabilities compared to those without disabilities. The study reported that persons with a disability were 1.22 times more likely to be unprepared for an emergency compared to persons without a disability (Smith and Notaro 2015). These results suggest that people with a disability may also be disproportionately at risk when facing natural and biological disasters.

In 2005, Hurricane Katrina demonstrated that people with medical needs, including both chronic diseases and disabilities, were subject to increased harm in the event of an emergency. After the hurricane, 31.5\% of Emergency Department visits during this disaster were related to chronic diseases (Sharma et al. 2008). Reasons for these visits included medication refills and maintenance for assistive technology or equipment for people with disabilities, and together these visits comprised more of the emergency department visits overall compared to visits due to injuries [29\%] (Sharma et al. 2008). While research related to the COVID-19 pandemic is still forthcoming, individuals with disabilities may have had increased disruptions in receiving prescriptions and other medical equipment and supplies during the pandemic (Alexander and Qato 2020). This evinces that chronic diseases and disabilities may both be risk factors for increased morbidity, mortality, and costs in the event of natural and biological disasters.

Emergency preparedness is a common concern among countries with studies comparing responses and programs in order to update and improve their reactions during a crisis (Kruk 2008; Moore et al. 2007; Shapira et al. 2020). However, despite international interest, the current literature is limited because it does not address the most vulnerable population of individuals who have both chronic disease and disability-i.e., the medically vulnerable. These individuals may be at the highest risk for morbidity due to the relationship between suffering from both chronic diseases and disabilities (Aldrich and Benson 2008). Exploring the combination of chronic diseases and disabilities can be of public health importance because it may increase the knowledge of emergency preparedness efforts for the most vulnerable populations, since individuals who believe that preparedness measures are important are more likely to adopt them (Wood et al. 2012). Thus, the purpose of this study was to evaluate the association between medically vulnerable populations and the status of emergency preparedness.

\section{Methods}

\section{Study design, population, and participants}

This cross-sectional study included a population-based sample of U.S. adults, selected from the 2010 and 2012 Behavioral Risk Factor Surveillance System (BRFSS). The BRFSS is a state-based surveillance system conducted by each state's health department in collaboration with the CDC (2020). It is a random-digit dialed telephone survey that aims to collect uniform, state-specific data on preventive health practices and risk behaviors in a non-institutionalized adult population (aged $\geq 18$ years) in all 50 states, as well as the District of Columbia and three U.S. territories (Puerto Rico, Guam, and the U.S. Virgin Islands) (CDC 2020). Trained interviewers collect data using a standardized questionnaire consisting of three parts: (1) core questions asked in all 50 states, the District of Columbia, and the three U.S. territories; (2) supplemental modules that a state can opt to include in its survey (e.g., questions on specific topics such as asthma history, mental health, or general preparedness); and (3) questions added by the states individually.

The Preparedness Module was included in the BRFSS as an optional module and was only used in 2010 and 2012 (CDC 2010, 2012). During those years, the states of Alabama, Montana, and North Carolina used this optional module. Thus, this analysis only considered study participants 
from these states $(n=35,547)$. Individuals were excluded if they were missing information on general preparedness $(n=$ 578). Additionally, individuals who were missing information for any of the five chronic conditions (diabetes $[n=23]$, stroke $[n=106]$, heart disease $[n=241]$, previous heart attack $[n=$ 127], and asthma [ $n=110])$, insurance status $(n=109)$, use of special equipment $(n=23)$, race/ethnicity $(n=235)$, marital status $(n=71)$ or education level $(n=49)$ were also excluded. Thus, 33,852 participants were available for analysis.

\section{Medically vulnerable assessment}

Research demonstrates that in the context of disasters, medically vulnerable populations are affected incommensurately to other populations (Bethel et al. 2011; Shapira et al. 2020). These individuals may be "at risk" and require more resources in disaster situations, with chronic health conditions playing a role. Previous research has typically categorized medically vulnerable as individuals with a disability or a chronic disease (Bethel et al. 2011). However, individuals with both a disability and a chronic disease may be more likely to suffer adverse outcomes during an emergency situation. Thus, this study classified medically vulnerable based on responses to chronic disease status and disability. This unique configuration represents a novel approach that attempts to address how disability and chronic disease may affect perceived preparedness.

In the core BRFSS standardized questionnaire, participants were asked "Have you ever been told by a doctor or nurse that you have any of the following: myocardial infarction (heart attack); stroke; chronic heart disease (CHD); asthma; or diabetes." Participants were classified as having a chronic disease if they responded "yes" to any of the above aforementioned chronic diseases. Additionally, participants responded to the question: "Do you now have any health problem that requires you to use special equipment, such as a cane, a wheelchair, a special bed, or a special telephone? (Include occasional use or use in certain circumstances)." Participants were classified as having a disability if they responded "yes" to the above question.

Based on the participants' responses, four groups were created. Group 1, labeled as "Medically Vulnerable," included participants classified as having both a disability and a chronic disease. Group 2, labeled as "Chronic Diseases," consisted of participants classified as having only a chronic disease while Group 3, labeled as "Disability," comprised participants classified as having any disability alone. Group 4, labeled "Referent group," included participants who did not have a chronic disease or a disability. This process was iterated individually for each chronic disease (i.e., history of heart attack, stroke, chronic heart disease, diabetes, and asthma).

\section{Preparedness assessment}

In the years 2010 and 2012, the states of Alabama, Montana, and North Carolina used the optional module on preparedness. To assess the general perceived preparedness outcome, participants were asked "How well prepared do you feel your household is to handle a large-scale disaster or emergency?". The responses for this question were "Well prepared," "Somewhat prepared," or "Not prepared at all." Individuals who responded "Well prepared" or "Somewhat prepared" were considered to have the outcome (i.e., preparedness). Participants who stated "Not prepared at all" were considered to not have the outcome.

\section{Assessment of potential confounders}

Sociodemographic variables such as race/ethnicity, age, sex, marital status, annual income, health insurance status, and education level were obtained from the BRFSS questionnaire and considered to be potential confounders of the medical vulnerability-preparedness association (Ablah et al. 2009; Bethel et al. 2011; Ko et al. 2014).

\section{Statistical analysis}

Frequencies and percentages were used to describe the sample's demographic characteristics, reported medical vulnerability, and preparedness. Logistic regression was used to assess the crude association between medical vulnerability and general preparedness. In addition, other factors associated with preparedness were identified. Multivariate logistic regression was used to obtain adjusted odds ratios (OR) and 95\% confidence intervals (CI). Final models included confounders that changed the magnitude of the relationship between the exposure and outcome by $10 \%$ or more (Maldonado and Greenland 1993). All analyses were conducted using SAS-callable SUDAAN to account for the complex sampling design of the BRFSS weighted sampling.

\section{Results}

The majority of participants were non-Hispanic white $(72.0 \%)$, married $(58.83 \%)$, females $(51.95 \%)$, ages 65 and older $(18.71 \%)$, and highly educated $(30.69 \%$ with a college education; Table 1). Nearly $86 \%$ of the total participants reported feeling prepared for a large-scale disaster or emergency. Medical vulnerability ranged from a high of $21.69 \%$ (asthma) to a low of $12.66 \%$ (heart attack). Asthma and stroke were chronic diseases with the highest frequencies $(15.79 \%$ and $13.48 \%$, respectively).

Participants who were non-Hispanic black ( $\mathrm{OR}=0.69$, $95 \%$ CI $0.60-0.80)$, Hispanic $(\mathrm{OR}=0.49,95 \%$ CI 0.36 - 
Table 1 Variables of respondents, BRFSS 2010 and 2012

Variables

Frequency (n)

Weighted \%

Total

33,852

100

Vulnerable 1

Heart attack and uses equipment

667

1.30

Heart attack does not use equipment

3.92

No heart attack and uses equipment

7.44

No heart attack and does not use equipment

2016

87.33

Vulnerable 2

$\mathrm{CHD}$ and uses equipment

CHD and does not use equipment

846

4.41

No CHD and uses equipment

1808

7.00

No CHD and does not use equipment

3192

86.84

Vulnerable 3

Stroke and uses equipment

28,006

3.35

Stroke and does not use equipment

1775

10.13

No stroke and uses equipment

5263

5.40

No stroke and does not use equipment

2263

81.12

Vulnerable 4

Asthma and uses equipment

2.85

Asthma and does not use equipment

1242

12.94

No asthma and uses equipment

4483

5.90

No asthma and does not use equipment

2796

78.31

Vulnerable 5

Diabetes and uses equipment

1295

2.84

Diabetes and does not equipment

3682

8.75

No diabetes and uses equipment

2743

5.91

No diabetes and does not use equipment

26,132

82.50

Race

Non-Hispanic white

27,132

72.00

Non-Hispanic black

3751

17.85

Hispanic

693

4.64

Other

2276

5.51

Sex

Male

12,980

48.05

Female

20,872

51.95

Education

Less than HS

3378

13.08

HS graduate

10,335

30.59

Some college

9408

28.91

College graduate

10,731

30.69

Marital status

Married

18,820

58.83

Divorced/separated

5590

11.83

Widowed

4931

6.53

Never married

3909

20.01

Member of unmarried couple

602

Age 
Table 1 (continued)

\begin{tabular}{|c|c|c|}
\hline Variables & Frequency (n) & Weighted \% \\
\hline $45-54$ & 6212 & 17.57 \\
\hline $55-64$ & 8103 & 18.37 \\
\hline$\geq 65$ & 11,104 & 18.71 \\
\hline \multicolumn{3}{|l|}{ Income } \\
\hline$<\$ 15,000$ & 4022 & 9.92 \\
\hline$\$ 15,000-\$ 25,000$ & 5877 & 15.87 \\
\hline$\$ 25,000-\$ 35,000$ & 3863 & 10.27 \\
\hline$\$ 35,000-\$ 50,000$ & 4720 & 12.52 \\
\hline$>\$ 50,000$ & 11,061 & 35.87 \\
\hline Do not know/missing & 4309 & 15.54 \\
\hline \multicolumn{3}{|l|}{ Health insurance status } \\
\hline Yes & 29,448 & 81.38 \\
\hline No & 4404 & 18.62 \\
\hline \multicolumn{3}{|l|}{ States } \\
\hline Alabama & 14,488 & 58.32 \\
\hline North Carolina & 11,162 & 29.34 \\
\hline Montana & 8202 & 12.33 \\
\hline \multicolumn{3}{|l|}{ General preparedness } \\
\hline Yes & 28,834 & 85.23 \\
\hline No & 5018 & 14.77 \\
\hline
\end{tabular}

0.66), or other with regard to race/ethnicity $(\mathrm{OR}=0.63,95 \%$ CI $0.44-0.82$ ) had approximately $40 \%$ to $50 \%$ decreased odds of being prepared compared to non-Hispanic white participants (Table 2). Males had approximately 1.4 times the odds of being prepared compared to females (95\% CI 1.22-1.55). Individuals who were considered the most medically vulnerable (those who had a chronic condition and a disability) had a $36 \%$ to $39 \%$ decreased odds of being prepared compared to individuals in the referent group, and these findings were statistically significant for all chronic conditions except for a history of heart attack (most medically vulnerable, CHD: $\mathrm{OR}=0.61,95 \%$ CI $0.46-0.82$, most medically vulnerable, stroke: $\mathrm{OR}=0.63,95 \%$ CI $0.51-0.77$, most medically vulnerable, asthma: $\mathrm{OR}=0.64,95 \%$ CI $0.50-0.81$, most medically vulnerable, diabetes: $\mathrm{OR}=0.62,95 \%$ CI 0.47-0.80).

After adjusting for education, income, and age, the magnitude of the associations for the most vulnerable category (chronic disease and disability requiring the use of assistive equipment) and preparedness remained largely unchanged (Table 3). Specifically, medically vulnerable individuals had statistically significant decreased odds of preparedness for nearly all of the chronic conditions investigated compared to those who did not have a history of the five chronic conditions combined with the use of special equipment (CHD OR 0.61, 95\% CI 0.46-0.82, stroke OR 0.63, 95\% CI 0.51-0.77, asthma OR 0.54, 95\% CI 0.43-0.69, and diabetes OR $0.6195 \%$ CI 0.48-0.77; Table 3). While individuals who were medically vulnerable for the heart attack condition also had decreased odds of preparedness, this finding was not statistically significant (OR 0.73 , 95\% CI 0.50-1.06). Individuals who had a chronic disease alone, without the use of assistive equipment, also had decreased odds of preparedness; however, these results were only significant for heart attack and CHD (OR 0.79, 95\% CI 0.66-0.93 and OR 0.81, 95\% CI 0.67-0.97, respectively).

\section{Discussion}

\section{Main findings of this study}

The purpose of this study was to evaluate the association between medically vulnerable populations and emergency preparedness. After adjustment for confounders, medically vulnerable individuals for all the conditions studied had decreased odds of general preparedness compared to individuals without chronic disease or disability.

\section{What is already known on this topic}

The results of this study are consistent with the literature indicating that, despite being the most vulnerable and often having distinctive needs that might require more time and planning in the event of a disaster (Federal Emergency 
Table 2 Odds ratios and 95\% CI associations between selected SES variables and preparedness, 2010 and 2012 BRFSS

\begin{tabular}{|c|c|c|c|}
\hline Variables & OR & Lower $95 \%$ CI & Upper $95 \%$ CI \\
\hline \multicolumn{4}{|l|}{ Vulnerable 1} \\
\hline Heart attack and uses equipment & 0.74 & 0.51 & 1.06 \\
\hline Heart attack does not use equipment & 1.11 & 0.86 & 1.44 \\
\hline No heart attack and uses equipment & 0.79 & 0.67 & 0.93 \\
\hline No heart attack and does not use equipment (referent) & 1.00 & 1.00 & 1.00 \\
\hline \multicolumn{4}{|l|}{ Vulnerable 2} \\
\hline CHD and uses equipment & 0.61 & 0.46 & 0.81 \\
\hline CHD and does not use equipment & 0.86 & 0.69 & 1.06 \\
\hline No CHD and uses equipment & 0.82 & 0.69 & 0.98 \\
\hline No CHD and does not use equipment (referent) & 1.00 & 1.00 & 1.00 \\
\hline \multicolumn{4}{|l|}{ Vulnerable 3} \\
\hline Stroke and uses equipment & 0.64 & 0.52 & 0.78 \\
\hline Stroke and does not use equipment & 0.92 & 0.79 & 1.07 \\
\hline No stroke and uses equipment & 0.87 & 0.80 & 1.08 \\
\hline No stroke and does not use equipment (referent) & 1.00 & 1.00 & 1.00 \\
\hline \multicolumn{4}{|l|}{ Vulnerable 4} \\
\hline Asthma and uses equipment & 0.54 & 0.43 & 0.69 \\
\hline Asthma and does not use equipment & 0.70 & 0.60 & 0.82 \\
\hline No asthma and uses equipment & 0.87 & 0.72 & 1.05 \\
\hline No asthma and does not use equipment (referent) & 1.00 & 1.00 & 1.00 \\
\hline \multicolumn{4}{|l|}{ Vulnerable 5} \\
\hline Diabetes and uses equipment & 0.61 & 0.48 & 0.77 \\
\hline Diabetes and does not equipment & 1.05 & 0.88 & 1.25 \\
\hline No diabetes and uses equipment & 0.89 & 0.73 & 1.08 \\
\hline No diabetes and does not use equipment (referent) & 1.00 & 1.00 & 1.00 \\
\hline \multicolumn{4}{|l|}{ Race } \\
\hline Non-Hispanic white (referent) & 1.00 & 1.00 & 1.00 \\
\hline Non-Hispanic black & 0.69 & 0.60 & 0.80 \\
\hline Hispanic & 0.49 & 0.36 & 0.66 \\
\hline Other & 0.63 & 0.44 & 0.82 \\
\hline \multicolumn{4}{|l|}{ Sex } \\
\hline Male & 1.38 & 1.22 & 1.55 \\
\hline Female (referent) & 1.00 & 1.00 & 1.00 \\
\hline \multicolumn{4}{|l|}{ Education } \\
\hline Less than HS & 0.38 & 0.32 & 0.45 \\
\hline HS graduate & 0.71 & 0.61 & 0.82 \\
\hline Some college & 0.68 & 0.59 & 0.80 \\
\hline College graduate (referent) & 1.00 & 1.00 & 1.00 \\
\hline \multicolumn{4}{|l|}{ Marital status } \\
\hline Married (referent) & 1.00 & 1.00 & 1.00 \\
\hline Divorced/separated & 0.60 & 0.53 & 0.69 \\
\hline Widowed & 1.10 & 0.93 & 1.30 \\
\hline Never married & 0.72 & 0.61 & 0.86 \\
\hline Member of unmarried couple & 0.56 & 0.38 & 0.81 \\
\hline \multicolumn{4}{|l|}{ Age } \\
\hline $18-24$ & 0.52 & 0.40 & 0.68 \\
\hline $25-34$ & 0.48 & 0.40 & 0.58 \\
\hline $35-44$ & 0.43 & 0.37 & 0.51 \\
\hline $45-54$ & 0.61 & 0.53 & 0.71 \\
\hline
\end{tabular}


Table 2 (continued)

\begin{tabular}{|c|c|c|c|}
\hline Variables & OR & Lower $95 \% \mathrm{CI}$ & Upper $95 \% \mathrm{CI}$ \\
\hline $55-64$ & 0.73 & 0.63 & 0.85 \\
\hline$\geq 65$ (referent) & 1.00 & 1.00 & 1.00 \\
\hline \multicolumn{4}{|l|}{ Income } \\
\hline$<\$ 15,000$ & 0.34 & 0.29 & 0.40 \\
\hline$\$ 15,000-\$ 25,000$ & 0.44 & 0.37 & 0.52 \\
\hline$\$ 25,000-\$ 35,000$ & 0.57 & 0.46 & 0.70 \\
\hline$\$ 35,000-\$ 50,000$ & 0.74 & 0.60 & 0.90 \\
\hline$>\$ 50,000$ (referent) & 1.00 & 1.00 & 1.00 \\
\hline Do not know/missing & 0.84 & 0.68 & 1.03 \\
\hline \multicolumn{4}{|l|}{ State } \\
\hline North Carolina (referent) & 1.00 & 1.00 & 1.00 \\
\hline Montana & 1.27 & 1.14 & 1.42 \\
\hline Alabama & 1.16 & 1.04 & 1.31 \\
\hline \multicolumn{4}{|l|}{ Health insurance status } \\
\hline Yes (referent) & 1.00 & 1.00 & 1.00 \\
\hline No & 0.59 & 0.48 & 0.72 \\
\hline
\end{tabular}

Table 3 Adjusted odds ratios of medically vulnerable and preparedness, BRFSS 2010 and 2012

\begin{tabular}{ll}
\hline Variables & Adjusted OR (95\% CI) \\
\hline Vulnerable 1 & \\
Heart attack and uses equipment & $0.73(0.50-1.06)$ \\
Heart attack does not use equipment & $0.94(0.73-1.22)$ \\
No heart attack and uses equipment & $0.79(0.66-0.93)$ \\
No heart attack and does not use equipment (referent) & 1.00 \\
Vulnerable 2 & \\
CHD and uses equipment & $0.61(0.46-0.82)$ \\
CHD and does not use equipment & $0.78(0.62-0.97)$ \\
No CHD and uses equipment & $0.81(0.67-0.97)$ \\
No CHD and does not use equipment (referent) & 1.00 \\
Vulnerable 3 & \\
Stroke and uses equipment & $0.63(0.51-0.77)$ \\
Stroke and does not use equipment & $0.87(0.74-1.01)$ \\
No stroke and uses equipment & $0.86(0.69-1.07)$ \\
No stroke and does not use equipment (referent) & 1.00 \\
Vulnerable 4 & \\
Asthma and uses equipment & $0.64(0.50-0.81)$ \\
Asthma and does not use equipment & $0.81(0.70-0.95)$ \\
No asthma and uses equipment & $0.83(0.68-1.01)$ \\
No asthma and does not use equipment (referent) & 1.00 \\
Vulnerable 5 & \\
Diabetes and uses equipment & $0.62(0.47-0.80)$ \\
Diabetes and does not equipment & $0.93(0.77-1.11)$ \\
No diabetes and uses equipment & $0.87(0.72-1.05)$ \\
No diabetes and does not use equipment (referent) & 1.00 \\
& \\
\hline
\end{tabular}


Management Agency [FEMA] 2004), people who have chronic diseases and disabilities do not perceive themselves to be any more prepared for an emergency than people without chronic diseases and disabilities (Bethel et al. 2011; Ko et al. 2014). For example, Bethel et al. (2011) used 2006-2008 BRFSS data from select states and demonstrated that individuals with fair/poor perceived health, a disability, and three or more chronic diseases had decreased odds of reporting that they had household preparedness items compared to individuals who were healthier. While the current study's results are congruent with previous studies, it is important to note that the current study considered chronic diseases combined with a disability while previous studies considered chronic diseases alone or disabilities alone (Smith and Notaro 2015; Bethel et al. 2011). It is possible that those who are "most vulnerable" have additional challenges because they have a history of a major medical condition in addition to requiring the use of special equipment. This may be a factor when responding as to whether they perceived themselves prepared or not; it is plausible that those who are most vulnerable have a greater time burden placed on them when performing day-to-day activities as a result of their condition, leaving less time for preparedness. Conversely, those who are most vulnerable could have a heightened sense of needing to be prepared but feel psychologically "less prepared."

\section{Limitations of this study}

This study has multiple limitations and strengths. Selection bias cannot be ruled out because the response rate of BRFSS was relatively low in both 2010 and 2012, during which the median response rates were $54.6 \%$ and $45.2 \%$, respectively (CDC 2010, 2012). Despite the low response rates, the BRFSS has response rates comparable to similar surveys (CDC 2012). Nondifferential misclassification may have occurred for both the exposure and outcome. The exposure, medical vulnerability, was categorized using self-reported responses to multiple questions. Since the BRFSS does not obtain detailed medical histories, survey data may not accurately reflect the breadth of these major medical conditions and their severity or impact on individuals. Likewise, respondents' use of special equipment may be unrelated to their medical conditions. In addition, individuals may not have wanted to disclose that they required additional assistance. In regard to the outcome, it is important to note that BRFSS asked only one question on perceived preparedness, and this particular question was only selected to be part of the survey by a few states in 2010 and 2012. Likewise, social desirability may have contributed to individuals answering that they were more prepared for emergencies. If this type of misclassification occurred, it would likely bias the results toward the null. The possibility of information bias was reduced since trained interviewers conducted the survey. While recognizing the preparedness question was only asked by a few states in 2010 and 2012, given the large sample size and complex sampling design used by BRFSS, the results may be generalized to the states analyzed in this study and possibly to the entire U.S. population.

\section{What this study adds}

This study has significant implications for the global public health community, particularly given the COVID-19 pandemic. To our knowledge, this is the first study to combine disability (as defined by the use of special equipment) and the presence of chronic conditions in the context of emergency preparedness. As both the severity and frequency of adverse events increase due to factors such as climate change and the emergence of new infectious disease including COVID-19, further research in this area will be warranted. This is particularly important given that the preparedness module has not been included as part of the BRFSS since 2012. Public health professionals should direct their efforts toward medically vulnerable individuals and their preparedness statuses. Disseminating educational materials to medically vulnerable populations may be beneficial for increasing their preparedness efforts. This study further solidifies the need for partnerships between medical, emergency, and public health personnel.

Authors' contributions Authors contributed equally to this work.

Funding No funding was received to assist with the preparation of this manuscript.

Data availability The data that support the findings of this study are available at the website of the Centers for Disease Control and Prevention at https://www.cdc.gov/brfss/index.html

Code availability Not applicable.

\section{Declarations}

Ethics approval The authors declare the study was done in compliance with Ethical Standards.

Informed consent During data collection, BRFSS personnel contacted potential participants by phone. During this phone call, participants gave verbal consent to participate. Participants were also informed that answers to the BRFSS questionnaire were confidential and that they did not need to respond to all questions and could end the interview at any time.

Conflict of interest The authors declare that they have no conflict of interest.

Research involving human subjects This research used publicly available, de-identified data that can be downloaded without restriction from the BRFSS website (https://www.cdc.gov/brfss/data_documentation/ 
index.htm). The authors' institution recognizes that the analysis of deidentified, publicly available data does not constitute human subjects research as defined in federal regulations, and as such does not require IRB review.

\section{References}

Ablah E, Konda K, Kelley CL (2009) Factors predicting individual emergency preparedness: a multi-state analysis of 2006 BRFSS data. Biosecur Bioterror 7(3):317-330. https://doi.org/10.1089/bsp. 2009.0022

Aldrich N, Benson WF (2008) Disaster preparedness and the chronic disease needs of vulnerable older adults. Prev Chronic Dis 5(1):A27

Alexander GC, Qato DM (2020) Ensuring access to medications in the US during the COVID-19 pandemic. JAMA 324(1):31-32. https:// doi.org/10.1001/jama.2020.6016

Bethel JW, Foreman AN, Burke SC (2011) Disaster preparedness among medically vulnerable populations. Am J Prev Med 40(2):139-143. https://doi.org/10.1016/j.amepre.2010.10.020

Bureau of Labor Statistics U.S. Department of Labor (2020) Unemployment rate rises to record high 14.7 percent in April 2020. https://www.bls.gov/opub/ted/2020/unemployment-raterises-to-record-high-14-point-7-percent-in-april-2020.htm. Accessed 3 June 2020

Centers for Disease Control and Prevention (2010) Behavioral Risk Factor Surveillance System 2010 Summary Data Quality Report. https://www.cdc.gov/brfss/annual_data/2010/pdf/2010_Summary_ Data_Quality_Report.pdf. Accessed 3 June 2020

Centers for Disease Control and Prevention (2012) Behavioral Risk Factor Surveillance System 2012 Summary Data Quality Report. https://www.cdc.gov/brfss/annual data/2012/pdf/ SummaryDataQualityReport2012_2013071ㄹ.pdf. Accessed 3 June 2020 last date accessed.

Centers for Disease Control and Prevention (2015) CDC: 53 million adults in the US live with a disability. https://www.cdc.gov/media/ releases/2015/p0730-US-disability.html. Accessed 15 June 2020

Centers for Disease Control and Prevention (2020) Behavioral Risk Factor Surveillance System (BRFSS). https://www.cdc.gov/brfss/ index.html. Accessed 2 June 2020

Federal Emergency Management Agency (2004) Preparing for disaster for people with disabilities and other special needs

Guha-Sapir D, Vos F, Below R, Ponserre S (2012) Annual disaster statistical review 2011: the numbers and trends. Centre for Research on the Epidemiology of Disasters (CRED), Brussels

Johns Hopkins University and Medicine (2021) Coronavirus Resource Center. https://coronavirus.jhu.edu. Accessed 6 Jan 2021

Ko JY, Strine TW, Allweiss P (2014) Chronic conditions and household preparedness for public health emergencies: Behavioral risk factor surveillance system, 2006-2010. Prehosp Disaster Med 29(1):1320. https://doi.org/10.1017/S1049023X13009126

Kruk ME (2008) Emergency preparedness and public health systems lessons for developing countries. Am J Prev Med 34(6):529-534. https://doi.org/10.1016/j.amepre.2008.02.012
Maldonado G, Greenland S (1993) Simulation study of confounderselection strategies. Am J Epidemiol 138(11):923-936. https://doi. org/10.1093/oxfordjournals.aje.a116813

Moore S, Mawji A, Shiell A, Noseworthy T (2007) Public health preparedness: a systems-level approach. J Epidemiol Community Health 61(4):282-286. https://doi.org/10.1136/jech.2004.030783

National Center for Immunization and Respiratory Diseases (NCIRD) (2020) Division of Viral Diseases Coronavirus Disease 2019 (COVID-2019). Retrieved from: https://www.cdc.gov/coronavirus/ 2019-ncov/cases-updates/cases-in-us.html2019-ncov/casesupdates/cases-in-us.html. Accessed 5 Jan 2021

National Oceanic and Atmospheric Administration (NOAA) (2020) National Centers for Environmental Information (NCEI) U.S. Billion-Dollar Weather and Climate Disasters. https:// www.ncdc.noaa.gov/billions/

Nick GA, Savoia E, Elqura L, Crowther MS, Cohen B, Leary M, Wright T, Auerbach J, Koh HK (2009) Emergency preparedness for vulnerable populations: people with special health-care needs. Public Health Rep 124(2):338-343. https://doi.org/10.1177/ 003335490912400225

O'Sullivan TL, Phillips KP (2019) From SARS to pandemic influenza: the framing of high-risk populations. Nat Hazards (Dordr) 98(1): 103-117. https://doi.org/10.1007/s11069-019-03584-6

Shapira S, Aharonson-Daniel L, Clarfield AM, Feder-Bubis P (2020) Giving a voice to medically vulnerable populations: a mixedmethods investigation of their unique perceptions and needs in emergency situations. Health Soc Care Community 28(3):811822. https://doi.org/10.1111/hsc.12911

Sharma AJ, Weiss EC, Young SL, Stephens K, Ratard R, StraifBourgeois S, Sokol TM, Vranken P, Rubin CH (2008) Chronic disease and related conditions at emergency treatment facilities in the New Orleans area after hurricane Katrina. Disaster Med Public Health Prep 2(1):27-32. https://doi.org/10.1097/DMP. ob013e31816452fo

Smith DL, Notaro SJ (2015) Is emergency preparedness a 'disaster' for people with disabilities in the US? Results from the 2006-2012 Behavioral risk factor surveillance system (BRFSS). Disability \& Society 30:401-418

United Nations [UN] (2009) UNISDR terminology for disaster risk reduction. https://www.unisdr.org/files/ 7817 UNISDRTerminologyEnglish.pdf. Accessed 3 June 2020

Ward BW, Schiller JS, Goodman RA (2014) Multiple chronic conditions among US adults: a 2012 update. Prev Chronic Dis 11:130389. https://doi.org/10.5888/pcd11.130389

Wood MM, Mileti DS, Kano M, Kelley MM, Regan R, Bourque LB (2012) Communicating actionable risk for terrorism and other hazards. Risk Anal 32(4):601-615. https://doi.org/10.1111/j.15396924.2011.01645.x

Publisher's note Springer Nature remains neutral with regard to jurisdictional claims in published maps and institutional affiliations. 